\title{
Utilization of unlocked nucleic acid (UNA) to enhance siRNA performance in vitro and in vivo
}

\author{
Maria B. Laursen, $\dagger^{a g}$ Malgorzata M. Pakula, $\dagger^{a b}$ Shan Gao, ${ }^{a b}$ Kees Fluiter, ${ }^{d}$ \\ Olaf R. Mook, ${ }^{d}$ Frank Baas, ${ }^{e}$ Niels Langklar, ${ }^{c}$ Suzy L. Wengel, ${ }^{c}$ Jesper Wengel, ${ }^{c}$ \\ Jørgen Kjems $^{a b}$ and Jesper B. Bramsen $* b$
}

Received 11th September 2009, Accepted 22nd December 2009

First published as an Advance Article on the web 9th February 2010

DOI: $10.1039 / \mathbf{b} 918869 j$

Small interfering RNAs (siRNAs) are now established as a favourite tool to reduce gene expression by RNA interference (RNAi) in mammalian cell culture. However, limitations in potency, duration, delivery and specificity of the gene knockdown (KD) are still major obstacles that need further addressing. Recent studies have successfully improved siRNA performance by the introduction of several types of chemical modifications. Here we explore the effect of incorporating unlocked nucleic acid (UNA) into siRNA designs. The acyclic UNA monomers lack the $\mathrm{C}^{\prime}$ - $\mathrm{C} 3^{\prime}$-bond of the RNA ribose ring and additively decrease nucleic acid duplex thermostability. We show that UNA-modifications of siRNAs are compatible with efficient RNAi and can improve siRNA performance both in vitro and in vivo. In particular, we find that the destabilizing properties of UNA are well suited to enhance the potency of siRNAs which are heavily modified by other chemical modifications such as locked nucleic acid (LNA), C4'hydroxymethyl-DNA (HM), 2'-O-methyl-RNA (OMe), DNA and 2'-Flouro-DNA (F). Interestingly, we find that naked, but UNA-modified siRNAs have dramatically increased biostability in mice and can induce potent KD in a xenograft model of human pancreas cancer. Hereby UNA constitutes an important type of chemical modification for future siRNA designs.

\section{Introduction}

The use of siRNA to knock down gene function has truly revolutionized mammalian cell cultures studies and holds great promise in therapeutics. ${ }^{1-3}$ Several siRNA designs have been suggested, ${ }^{4,5}$ yet most studies utilizes a standard siRNA design comprising a 21 base pair (bp) RNA duplex containing a 19 mer base pairing stem and 2 nucleotide (nt) $3^{\prime}$ overhangs. ${ }^{6}$ Upon introduction into the cell cytoplasm siRNAs are taken up into the RNA-induced silencing complex (RISC), the nonguiding sense strand (SS) is degraded and the guiding antisense strand (AS) will subsequently direct the sequence-specific cleavage of perfectly complementary RNAs. ${ }^{7-13}$ In spite of the great potency and applicability of siRNAs, a number of obstacles still hamper their wider use. Most prominent is the

${ }^{a}$ Department of Molecular Biology, Aarhus University,

DK-8000 Aarhus, Denmark

${ }^{b}$ Interdiciplinary Nanoscience Center, Aarhus University,

DK-8000 Aarhus,Denmark.E-mail:jebb@mb.au.dk;

Fax: + 458619 6500; Tel: +458942 2668

${ }^{c}$ Nucleic Acids Center, Department of Chemistry,

University of Southern Denmark, DK-5230 Odense M, Denmark

${ }^{d}$ Macrozyme BV, Meibergdreef 45, 1105 BA Amsterdam,

the Netherlands

${ }^{e}$ Dept of Neurogenetics, AMC, Meibergdreef 9, 1105 AZ Amsterdam, the Netherlands

${ }^{f}$ Ribotask ApS, Promonadebyen 2-8, DK-5000 Odense C, Denmark

${ }^{g}$ Department of Haematology, Aalborg Hospital, Aarhus University

Hospital, DK-9000 Aalborg, Denmark

$\dagger$ Contributed equally to this work. current lack of efficient means of siRNA delivery under the harsh condition in vivo where naked siRNA are quickly degraded by extra- and intracellular ribonucleases. ${ }^{14,15}$ Other concerns are reports of poor KD specificity as siRNAs can both induce innate immune responses ${ }^{16}$ and exert broad off-target effects on non-intended targets sharing only partial sequence complementarity with the siRNA. ${ }^{17,18}$ To address these problem we and others have successfully modified siRNAs and identified highly active, more nuclease resistant and target-specific siRNAs. ${ }^{5,14,15,19-24}$ Recently, a large scale comparison of 21 types of chemical modifications in siRNA design found unlocked nucleic acid (UNA) to have several interesting properties. $^{23}$ UNA monomers are acyclic derivatives of RNA lacking the $\mathrm{C} 2{ }^{\prime}-\mathrm{C}^{\prime}$ '-bond of the RNA ribose ring, yet still structurally mimicking unmodified RNA upon incorporation into RNA duplexes. Incorporation of UNA monomers into siRNAs induces additive instability by 5-8 ${ }^{\circ} \mathrm{C}$ per UNA monomer thereby providing a unique possibility to locally destabilize the siRNA duplex. ${ }^{25}$ Here we extend our analysis of incorporating UNA into siRNA designs and show how UNA can be utilized to improve siRNA performance. We find that low levels of UNA modifications in siRNAs are compatible with efficient RNAi whereas more extensive UNA modification lowers siRNA efficiency and serum stability. Yet, even low levels of UNA modification can be highly beneficial; especially the strong destabilizing property of UNA can be strategically used both in the SS and AS to significantly improve the potency of extensively 
modified siRNAs that are otherwise too stable or rigid to support RNAi. Interestingly, lightly UNA-modified siRNA has prolonged biostability in vivo, even compared to extensively LNA modified siRNA, and produces efficient gene KD in a mouse model bearing human pancreas tumour xenografts. Hereby, UNA constitutes an important type of siRNA modification that has several interesting properties for siRNA function both in vitro and in vivo.

\section{Results and discussion}

\section{Tolerance for UNA-modification in the SS}

To investigate the impact of UNA-modification on siRNA activity we generated a H1299 cell line stably expressing a dual luciferase reporter harbouring a single perfect eGFP target site within the renilla luciferase $3^{\prime} \mathrm{UTR}$. This cell line was transfected with chemically modified siRNAs at either 1 or $10 \mathrm{nM}$ concentration (final concentration in the medium) using the Lipofectamine 2000 transfection reagent and the relative KD of renilla luciferase activity was evaluated $48 \mathrm{~h}$ after transfection (see Table 1 for an overview of the chemically modified ASs and SSs). We initially tested the tolerance for UNA modification at various positions within the SS or AS in combination with an unmodified opposing strand (Fig. 1A and 1B, respectively and see Fig. 1E for a structural representation of UNA). As reported for many other chemistries ${ }^{5}$ we find that single UNA modifications are well tolerated in the SS $3^{\prime}$ overhang (W131) and additionally also in the SS 5'end (W132), central region (W308), $3^{\prime}$ end (ID1276; Fig. 1A). Also triple UNA-modifications alone (W282) or in combination with 5'end LNA modification (W321) only reduced siRNA potency slightly as compared to the unmodified siRNA against eGFP, denoted siEGFP (Fig. 1A).

\section{Tolerance for UNA-modification in the AS}

We found that UNA-modification of the central part of the siRNA AS (W320, W126), 3'end (W125) and overhang (W127) are well tolerated, whereas UNA-modification of the AS seed region (position 2-8 of counting from the $5^{\prime}$ end) can lead to slightly reduced potency (W315, W317, W318; Fig. 1B). Also double and triple modifications within the base-pairing stem of the siRNA duplex (W329, W187, W281 and W186) resulted in reduced silencing efficiency (Fig. 1B). Since these ASs efficiently form siRNA duplexes upon annealing to the unmodified SS, W207 (Fig. 4A) we speculate that several UNA-modifications may excessively destabilize AS interactions with the target site.

\section{Tolerance for UNA-modification of siRNA duplexes}

We next combined the functional UNA-modified SSs and ASs to test the tolerance for simultaneous UNA-modification in both siRNA strands (Fig. 1C). As expected, siRNA efficiency was largely dependent on the activity of the UNA-modified AS; all single-modified ASs retained their high silencing activity in combination with all tested SSs irrespectively of the positioning of the UNA modifications within the AS and SS (see all combinations of ASs W317, W126, W125 with
SSs W132, W308, ID1278, W282; Fig. 1C). The activity of the double and triple UNA-modified ASs (W329, W187 and W186, respectively) were more sensitive to SS modification; in particular the silencing activity of these ASs declined in combination with the double UNA-modified SS, W282 (Fig. 1C). This reduction in silencing potency for extensively UNA-modified siRNAs may well be due to excessive thermodynamic destabilization of the siRNA duplex and indeed we were largely unable to anneal several of the highly modified siRNAs (such as W281-W308, W330-W282 and W281-W282; Fig. 4A). We therefore recommend not to incorporate more than two UNA modifications in total within the base-paring siRNA stem unless the siRNA is stabilized by other types of chemical modifications (see below).

\section{Combining UNA and LNA allow higher levels of siRNA modification}

As incorporation of more than one UNA modification into the AS resulted in reduced silencing (W329, W187, W186, W281; Fig. 1B) and reduced serum stability (Fig. 4A), we speculated that their potency may be enhanced in combination with a stabilizing, LNA-modified SS, W037. LNA monomers additively increase thermal stability by $2-10{ }^{\circ} \mathrm{C}$ per modification ${ }^{26}$ and thereby counteract siRNA destabilization by UNA. We have previously reported that the activity of 36 out of 48 chemically modified ASs was reduced in combination with the LNA modified SS, W037, as compared to an unmodified SS, W207, and we never found any improvement in AS potency by $\mathrm{W} 037 .^{23}$ Similarly, the potency of the unmodified AS (W053) is significantly reduced in combination with the LNA modified SS, W037 as compared to the unmodified SS, W207 (Fig. 3A). Here we found that the activity of both UNA modified AS, W329 and W187, was even enhanced in combination with W037 (Fig. 1D) and that the stability was greatly increased (stability is shown for W329 in Fig. 4B, data not shown for W187). This shows that balancing the use of the strongly stabilizing LNA and the destabilizing UNA can allow higher levels of siRNA modification and thereby enhance siRNA stability without compromising silencing efficiency.

\section{UNA-modification of SS $3^{\prime}$ end enhance siRNA performance}

As strand selection during RISC loading is dependent on the thermodynamic profile of the siRNA duplex ${ }^{27,28}$ we speculated that UNA destabilization of the SS $3^{\prime}$ end may enhance AS selection and thereby siRNA performance. To test this we generated a set of six ASs having single LNA-modifications at position 1-6 from the 5'end. As LNA-modifications are strongly stabilizing this generates a series of siRNA with a highly asymmetric thermodynamic profile unfavourable for AS incorporation into RISC. We found that UNA-modification at the $3^{\prime}$ double stranded end of the SS (ID1276) did indeed improve the performance of siRNAs having LNAmodifications at positions $1-3$ of the AS whereas no effect was seen for ASs modified at pos. 4-6 (Fig. 2A). This is in good agreement with previous studies showing that strand selection is primarily determined by the thermodynamic profile of the outermost positions of the stem. ${ }^{27,28} \mathrm{We}$ next asked if UNA-modification of the SS $3^{\prime}$ end may also improve 
A

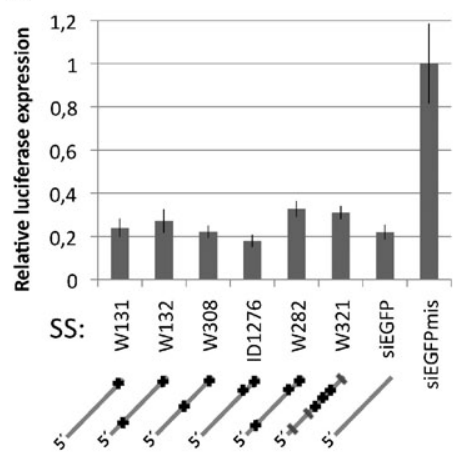

B

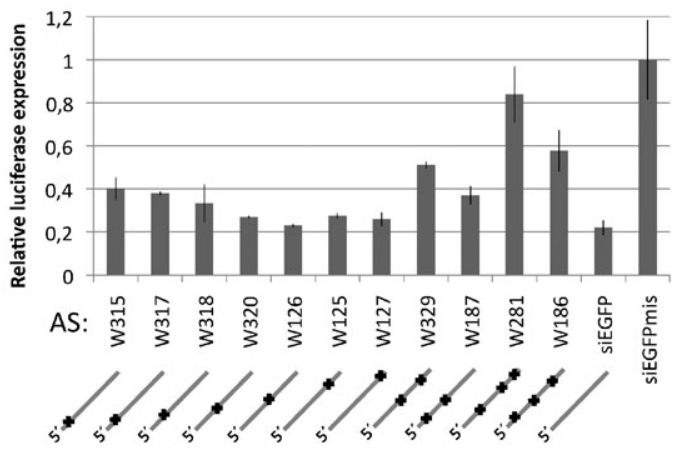

C

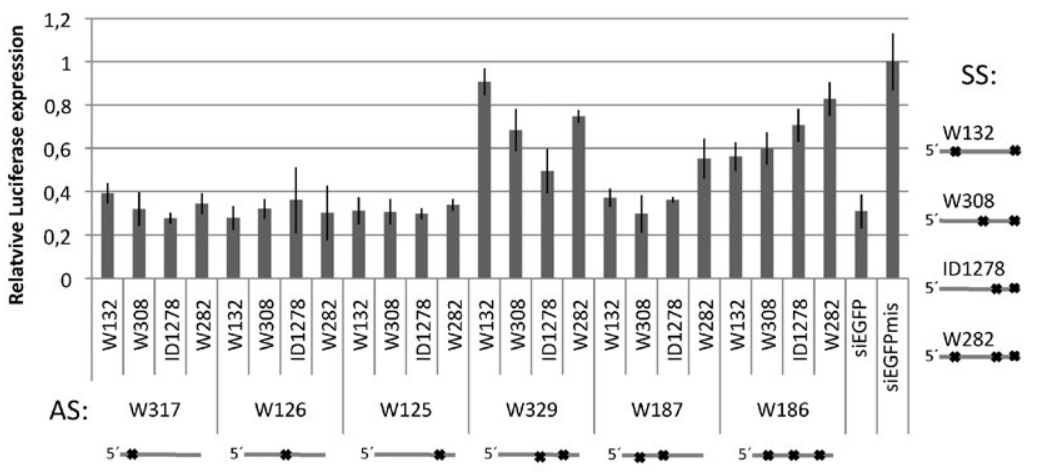

D

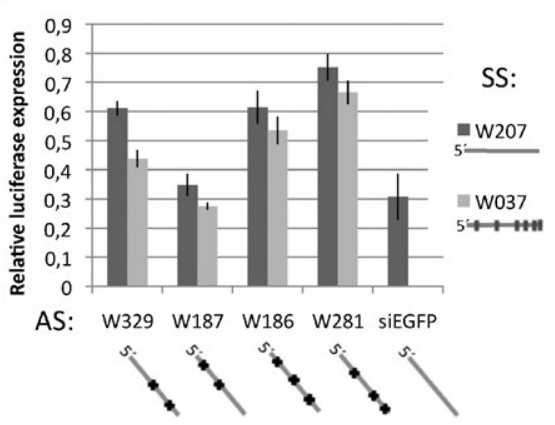

E

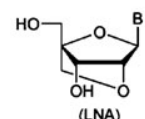

(LNA)

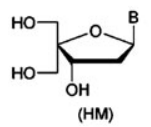

(HM)<smiles>[B]C1OC(CO)C(O)C1F</smiles>

列

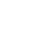


A

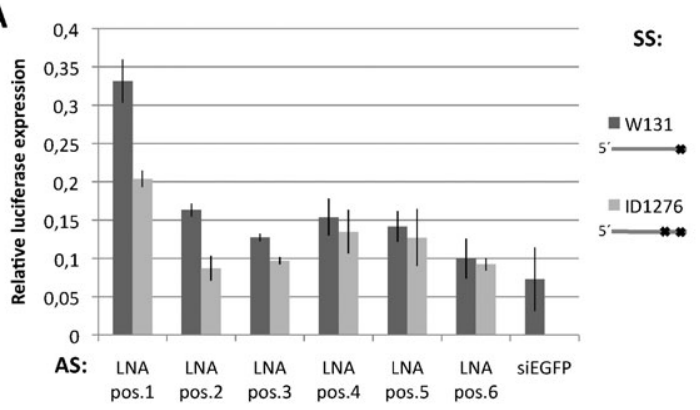

B

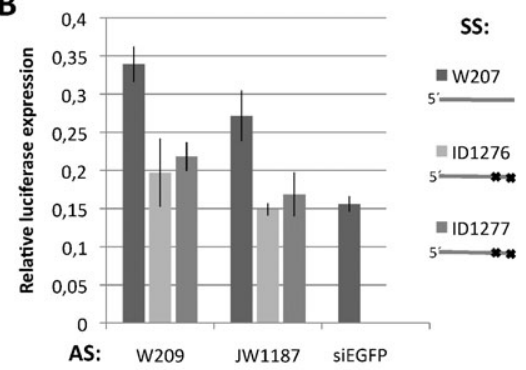

C

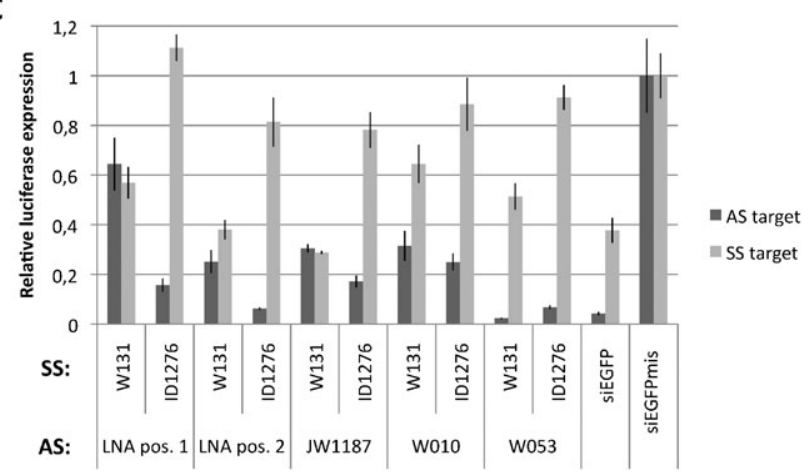

Fig. 2 UNA-modification of the SS 3'can improve siRNA performance by altering siRNA strand selection. (A) Silencing activity of ASs modified by LNA in position 1 to 6 counting from the AS $5^{\prime}$ end in combination with UNA-modified SSs either with or without UNA in the $3^{\prime}$ end (ID1276 and W131 respectively) (20 nM final concentration). (B) Silencing activity of extensively LNA- (W209) or HM-modified (JW1187) ASs in combinations with the unmodified SS W207 or SS modified by UNA in their 3'end (ID1276, ID1277) (10 nM final siRNA concentration). (C) Silencing activity of AS (on AS-sensor) and SS (on SS-sensor) of LNA- (pos. 1, pos. 2, W010) and HM-modified (JW1187) ASs in combination with UNA-modified SSs either with or without UNA in the $3^{\prime}$ end (ID1276 and W131, respectively) (20 nM final siRNA concentration). The position of the UNA modifications or LNA modifications are shown schematically right to the figures as black crosses (UNA) or grey lines (LNA), respectively. siEGFP is an unmodified siRNA duplex containing AS W053 and SS W207. siEGFPmis is a mismatch control.

W131 for all tested ASs whereas AS performance is improved (Fig. 2C). This strongly suggests that SS 3'end UNA-modification does indeed disfavour SS and thereby favour AS incorporation during RISC loading.

\section{UNA-modification of the SS enhance the activity of heavily modified siRNA duplexes}

We have previously reported that the so-called sisiRNA design characterized by a segmented LNA-modified SS enhances the potency of heavily modified siRNA likely by destabilizing or introducing flexibility into the siRNA duplex. ${ }^{5}$ Therefore, we speculated that thermodynamic destabilization imposed by the UNA modification would similarly introduce flexibility into extensive modified siRNAs and thereby enhance their potency. To test this we introduced two UNA-modifications into a heavily LNA-modified SS (compare W037 and NAC3168) and tested its impact on silencing in combinations with AS modified by OMe (Dharm1), alternating OMe/F (JC10), HM (JW1187) and LNA (W010) (see Table 1). Indeed we found that both the UNA-modified SS (NAC3168) and sisiRNA design (W004 + W179) enhanced the potency of most ASs (Dharm1, JC10, HM1187 and unmodified AS (W053) to levels comparable to the unmodified SS (W207; Fig. 3). Only the heavily LNA-modified AS (W010) were significantly more efficient in combination with the sisiRNA design
(W004 + W179) as compared to the UNA/LNA-modified SS (NAC3168), yet the silencing efficiency was still significantly improved by NAC3168 to levels indistinguishable from the unmodified SS, W207 (Fig. 3A). This shows that UNAmodification of the SS may be utilized in extensively modified siRNA designs to enhance silencing activities.

\section{UNA-modification of the AS can enhance the potency of heavily} modified siRNA duplexes

We next asked if UNA modifications could similarly be incorporated into ASs to enhance siRNA performance, especially of the heavily LNA-modified duplexes. To test this we inserted a central UNA modification into a heavily LNA-modified AS (W188) and combined these with the heavily LNA-modified SSs, W037 and W013. We found that the introduction of UNA-modification in the central part of AS did indeed enhance the efficiency of the siRNA duplexes for both LNA-modified SSs, W037 and W013 as compared to the LNA-modified AS without UNA (W010) (Fig. 3B). Furthermore, we generated two SSs fully substituted with mixed DNA and LNA modifications (W008, W009) and tested these in combination with an AS containing a central UNA modification, W126 (Fig. 3C). Again we found a higher performance of $\mathrm{W} 126$ as compared to the unmodified AS, W053. Collectively, this clearly demonstrates that a 
Table 1 Overview of chemically modified ASs and SSs. The name, type of chemical modification and sequence of the investigated ASs and SSs are given. The following modification abbreviations are used: 2'-O-methyl (OMe), 2'-fluoro (F), 2'-deoxy (DNA), 4'-C-hydroxymethyl-DNA (HM), locked nucleic acid (LNA), unlocked nucleic acid (UNA). The position of the modification is shown in bold in the oligo sequence. The oligoes are named according to the nomenclature of previously published work to allow easy comparison of results between individual studies

\begin{tabular}{|c|c|c|c|}
\hline Name & $\mathrm{AS} / \mathrm{SS}$ & Modification & Sequence $\left(5^{\prime}-3^{\prime}\right)$ \\
\hline Dharm 1 & AS & $\mathrm{OMe}$ & АС \\
\hline $\mathrm{JC} 10$ & AS & $\mathrm{F} / \mathrm{OMe}$ & $A_{F} C_{O M e} U_{F} U_{O M e} G_{F} U_{O M e} G_{F} G_{O M e} C_{F} C_{O M e} G_{F} U_{O M e} U_{F} U_{O M e} A_{F} C_{O M e} G_{F} U_{O M e} C_{F} G_{O M e} C_{F}$ \\
\hline JW1187 & AS & HM/LNA & $\mathrm{ACT}_{\mathbf{H M}} \mathrm{UGT}_{\mathbf{H M}} \mathrm{GGCCGUUT}_{\mathbf{H M}} \mathrm{ACGT}_{\mathbf{H M}} \mathrm{CG}_{\mathrm{LNA}} \mathbf{C}_{\mathbf{L N A}} \mathrm{U}$ \\
\hline W010 & AS & LNA & $\mathrm{ACT}_{\mathbf{L N A}} \mathrm{UGT}_{\mathbf{L N A}} \mathrm{GGCCGUUT}_{\mathbf{L N A}} \mathrm{ACGT}_{\mathbf{L N A}} \mathrm{CG}_{\mathbf{L N A}} \mathbf{C}_{\mathbf{L N A}} \mathrm{U}$ \\
\hline W053 & AS & & ACUUGUGGCCGUUUACGUCGC \\
\hline W095 & AS & ADA/LNA & ACUUGUGGCCGUUUACGT ${ }_{A D A} C_{G_{L N A}} C_{L N A} U$ \\
\hline W123 & AS & UNA/LNA & ACUUGU UNA $_{\mathbf{U N}}$ GGCCGUUUACGUCG $\mathbf{L N A}_{\mathbf{L N A}} \mathbf{C}_{\mathbf{L N}} \mathrm{U}$ \\
\hline W125 & AS & UNA/LNA & ACUUGUGGCCGUUUACGU $\mathbf{U N A}_{\mathbf{A}} \mathrm{CG}_{\mathrm{LNA}} \mathrm{C}_{\mathrm{LNA}} \mathrm{U}$ \\
\hline W126 & AS & UNA/LNA & ACUUGUGGCCGU ${ }_{\mathrm{UNA}} U \mathrm{UUACGUCG_{ \textrm {LNA } }} \mathbf{C}_{\mathrm{LNA}} \mathrm{U}$ \\
\hline W127 & AS & UNA & ACUUGUGGCCGUUUACGUCGC $\mathbf{U N A}_{\mathbf{A}} \mathrm{U}$ \\
\hline W128 & AS & UNA & 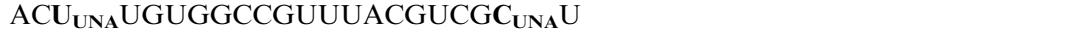 \\
\hline W180 & AS & LNA & ACUUGUGGCCGUUUACGUCG $\mathbf{L N A}_{\mathbf{L N A}}$ \\
\hline W186 & AS & UNA/LNA & ACUUGU $_{\mathbf{U N A}}$ GGCCGU $_{\mathbf{U N A}}$ UUACGU $\mathbf{U}_{\mathbf{U N A}} \mathbf{C G}_{\mathbf{L N A}} \mathbf{C}_{\mathbf{L N A}} \mathrm{U}$ \\
\hline W187 & AS & UNA/LNA & ACUUGU $_{\mathbf{U N A}}$ GGCCGU UNA $U$ UUAGUCG $\mathbf{L N A}_{\mathbf{L N A}} \mathbf{C}_{\mathbf{L N}}$ \\
\hline W188 & AS & UNA/LNA & $\mathrm{ACT}_{\mathbf{L N A}} \mathrm{UGT}_{\mathbf{L N A}} \mathrm{GGCCG}_{\mathbf{U N A}} \mathrm{UT}_{\mathbf{L N A}} \mathrm{ACGT}_{\mathbf{L N A}} \mathrm{CG}_{\mathbf{L N A}} \mathrm{C}_{\mathbf{L N A}} \mathrm{U}$ \\
\hline W209 & AS & LNA & $\mathrm{ACT}_{\mathbf{L N A}} \mathrm{UGT}_{\mathbf{L N A}} \mathrm{GGCCGUUT}_{\mathbf{L N A}} \mathrm{ACGT}_{\mathbf{L N A}} \mathrm{CG}_{\mathrm{LNA}} \mathbf{C}_{\mathbf{L N A}}$ \\
\hline W281 & AS & UNA & ACUUGUG $_{\mathbf{U N A}} \mathrm{GCCGUUUA}_{\mathbf{U N A}} \mathrm{CGUCGC}_{\mathbf{U N A}} \mathrm{U}$ \\
\hline W315 & AS & UNA/LNA & ACU $_{\mathbf{U N A}}$ UGUGGCCGUUUACGUCG $\mathbf{L N A}_{\mathbf{L A N A}} \mathbf{C}_{\mathbf{L N}} \mathrm{U}$ \\
\hline W317 & AS & UNA/LNA & ACUUG $_{U N A} U_{G G C C G U U U A C G U C G_{L N A}} \mathbf{C}_{\mathbf{L N A}} \mathrm{U}$ \\
\hline W318 & AS & UNA/LNA & ACUUGUG $_{U N A} G_{C C G U U U A C G U C G_{L N A}} \mathbf{C}_{\mathbf{L N A}} U$ \\
\hline W320 & AS & UNA/LNA & ACUUGUGGC $_{\mathbf{U N A}}$ CGUUUACGUCG $\mathbf{L N A}_{\mathbf{L N}} \mathbf{C}_{\mathbf{L N A}} \mathrm{U}$ \\
\hline W329 & AS & UNA/LNA & ACUUGUGGCCGU UNA $U$ UUACGU UNA $\mathrm{CG}_{\mathbf{L N A}} \mathbf{C}_{\mathbf{L N A}} \mathrm{U}$ \\
\hline W330 & AS & UNA & ACUUGUGGCCGU UNA UUACGUCGC $\mathbf{U N A}_{\mathbf{U N}} \mathrm{U}$ \\
\hline ID1276 & SS & UNA & 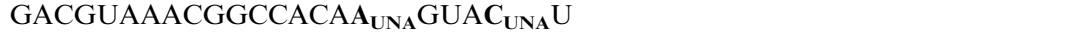 \\
\hline ID1277 & SS & UNA/LNA & GACGUAAACGGCCACAAG $_{\mathbf{U N A}} \mathrm{UT}_{\mathbf{L N A}} \mathrm{C}_{\mathbf{L N A}} \mathrm{U}$ \\
\hline ID1278 & SS & UNA & GACGUAAACGGCCACAAG $_{\mathbf{U N A}} \mathrm{UAC}_{\mathbf{U N A}} \mathrm{U}$ \\
\hline NAC3168 & SS & UNA/LNA & $\mathrm{GAC}_{\mathbf{L N A}} \mathrm{GUA}_{\mathbf{U N A}} \mathrm{AAC}_{\mathbf{L N A}} \mathrm{GGCC}_{\mathbf{L N A}} \mathrm{AC}_{\mathbf{L N A}} \mathrm{AA}_{\mathbf{U N A}} \mathrm{GUT}_{\mathbf{L N A}} \mathrm{C}_{\mathbf{L N A}} \mathrm{U}$ \\
\hline W004 + W179 & SS & LNA & GAC $_{\mathbf{L N A}}$ GUAAAC $\mathbf{L N A}_{\mathbf{L N}} \mathrm{G}+\mathrm{GCC}_{\mathbf{L N A}} \mathrm{AC}_{\mathbf{L N A}} \mathrm{AAGUT}_{\mathbf{L N A}} \mathrm{C}_{\mathbf{L N A}}$ \\
\hline W008 & SS & DNA/LNA & $\begin{array}{l}G_{D N A} A_{D N A} C_{L N A} G_{D N A} T_{D N A} A_{L N A} A_{D N A} A_{D N A} C_{L N A} G_{D N A} G_{D N A} C_{L N A} C_{D N A} A_{D N A} C_{L N A} A_{D N A} \\
A_{D N A} G_{L N A} T_{D N A} T_{L N A} C_{L N A} T_{D N A}\end{array}$ \\
\hline W009 & SS & DNA/LNA & $\begin{array}{l}G_{D N A} A_{L N A} C_{D N A} G_{L N A} T_{D N A} A_{L N A} A_{D N A} A_{D N A} C_{D N A} G_{L N A} G_{D N A} C_{L N A} C_{D N A} A_{L N A} C_{D N A} A_{L N A} \\
A_{D N A} G_{L N A} T_{D N A} T_{L N A} C_{L N A} T_{D N A}\end{array}$ \\
\hline W013 & SS & LNA & $\mathrm{GAC}_{\mathbf{L N A}} \mathrm{GT}_{\mathbf{L N A}} \mathrm{AA}_{\mathbf{L N A}} \mathrm{AC}_{\mathbf{L N A}} \mathrm{GGCCACAAGUT}_{\mathbf{L N A}} \mathbf{C}_{\mathbf{L N A}} \mathrm{U}$ \\
\hline W037 & SS & LNA & 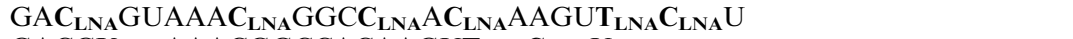 \\
\hline W129 & SS & UNA/LNA & GACGU $_{\mathbf{U N A}}$ AAACGGCCACAAGUT $\mathbf{L N A}_{\mathbf{L N A}} \mathbf{C}_{\mathbf{L N}} \mathrm{U}$ \\
\hline W130 & SS & UNA/LNA & GACGUAAACC $_{\mathbf{U N A}}$ GGCCACAAGUT $_{\mathbf{L N A}} \mathbf{C}_{\mathbf{L N A}} \mathrm{U}$ \\
\hline W131 & SS & UNA & GACGUAAACGGCCACAAGUUU $\mathbf{U N A} \mathrm{U}$ \\
\hline W132 & SS & UNA & 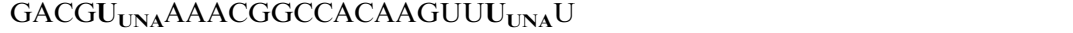 \\
\hline W181 & SS & LNA & GAC $_{\mathbf{L N A}} \mathrm{GUAAAC}_{\mathbf{L N A}} \mathrm{GGCC}_{\mathbf{L N A}} \mathrm{AC}_{\mathbf{L N A}} \mathrm{AAGUT}_{\mathbf{L N A}} \mathbf{C}_{\mathbf{L N A}} \mathrm{U}$ \\
\hline W282 & SS & UNA & GAC $_{\mathbf{U N A}}$ GUAAACGGCCACAA $\mathbf{A}_{\mathbf{U N A}} \mathrm{GUUU}_{\mathbf{U N A}} \mathrm{U}$ \\
\hline W308 & SS & UNA & GACGUAAACG $_{\mathbf{U N A}}$ GCCACAAGUUU UNA $U$ \\
\hline W321 & SS & UNA/LNA & GAC $_{\mathbf{L N A}}$ GUAAAC $_{\mathbf{L N A}} \mathbf{G}_{\mathrm{UNA}} \mathrm{GCC}_{\mathrm{UNA}} \mathrm{AC}_{\mathbf{U N A}} \mathrm{AAGUT}_{\mathbf{L N A}} \mathbf{C}_{\mathbf{L N A}} \mathrm{U}$ \\
\hline W207 & SS & & GACGUAAACGGCCACAAGUUC \\
\hline
\end{tabular}

UNA-modification of both the SS and ASs can significantly help increasing the number of total modifications that are tolerated in siRNA designs.

\section{The impact of UNA modification on siRNA biostability in vitro}

As UNA modification decreases siRNA thermodynamic stability, ${ }^{25}$ we next investigated the impact of UNA modification on siRNA biostability in vitro (Fig. 4A\&B) and upon injection of naked siRNAs in mice (Fig. 5A\&B). Notably, we were unable to anneal the most heavily UNA-modified siRNA duplexes such as W330-W282, W281-W282 and W281-W308 (Fig. 4A) suggesting that care must be taken when incorporating more than two UNA-modifications into the stem of siRNAs. Furthermore all investigated siRNAs modified exclusively by UNA within the stem had significantly reduced stability and were largely degraded after $5 \mathrm{~min}$ of incubation in $80 \%$ FCS (Fig. 4A). We therefore recommend the use of shielding delivery reagents when utilizing siRNA modified only by UNA within the base pairing stem. In contrast, insertion of UNA into the stem of very extensively modified siRNA such as $\mathrm{OMe} / \mathrm{F} / \mathrm{UNA}$-modified and LNA-modified duplexes did not significantly reduce serum stability (compare JC10-W037 to JC10-NAC3168 and W209-ID1276 to W209-W207, respectively; Fig. 4B). Furthermore, the incorporation of UNA in either the SS or AS of extensively LNA-modified siRNA only marginally reduced siRNA serum stability (compare W010-NAC3168 and W188-W037 to W010-W037; Fig. 4B) although siRNA potency was significantly enhanced (Fig. 3).

\section{The impact of UNA modification on siRNA biostability and knock down efficiency in vivo}

We have previously observed that extensive LNA-stabilization can prolong the blood circulation time of naked siRNA in 


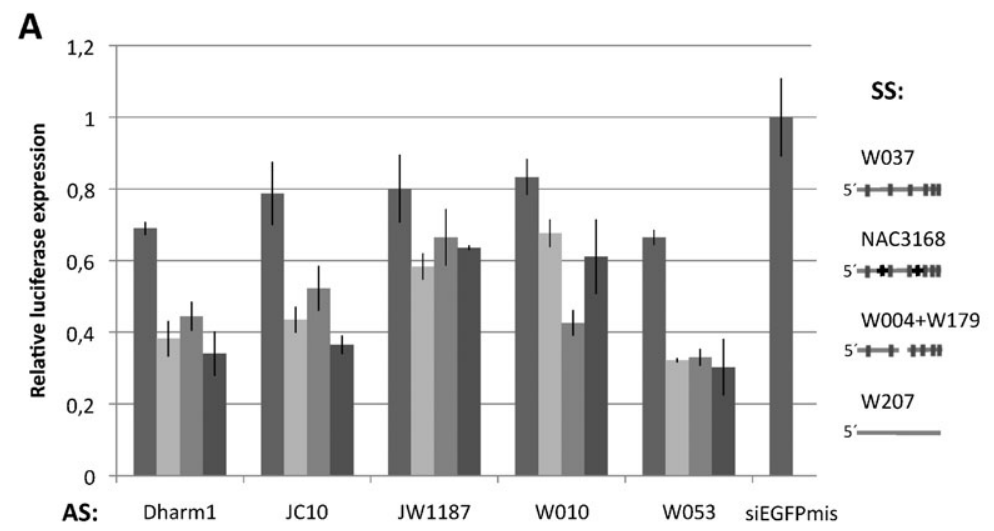

B

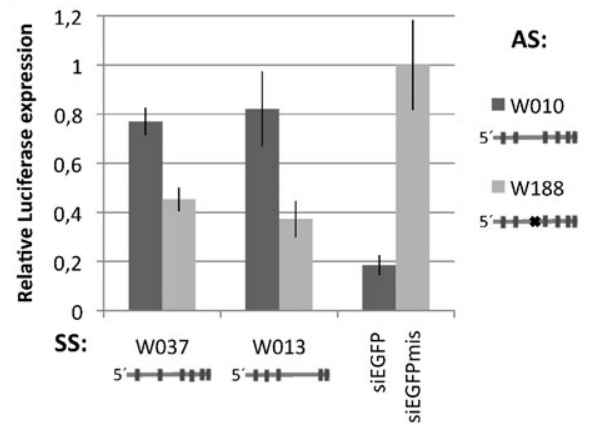

C

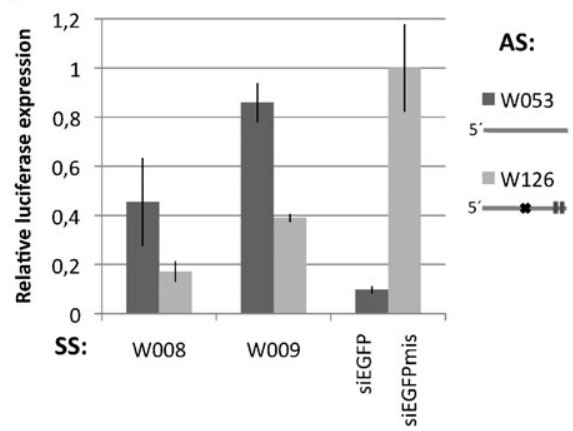

Fig. 3 UNA-modification of ASs and SSs can enhance the potency of extensively modified siRNAs. A. Silencing activity of ASs modified by OMe (Dharm1), OMe/F (JC10), HM (JW1187), LNA (W010) in combination with either an extensively LNA modified SS (W037), segmented SS (W004 + W179), UNA/LNA-modified SS (NAC3168) or unmodified SS (W207) (1 nM final siRNA concentration). B. Silencing activity of extensively LNA-modified (W010) or LNA/UNA-modified (W188) ASs in combination with extensively LNA-modified SSs, W037 or W013 (10 nM final siRNA concentration). C. Silencing activity of unmodified AS (W053) and UNA modified (W126) AS in combination with fully DNA-LNA substituted SSs (W008, W009) (10 nM final siRNA concentration). The position of the UNA modifications or LNA modifications are shown schematically below or right to the figures as black crosses (UNA) or grey lines (LNA), respectively. siEGFP is an unmodified siRNA duplex containing the AS W053 and SS W207. siEGFPmis is a mismatch control.

mice upon intravenous injection ${ }^{29}$ and mediate efficient KD of eGFP in the lungs. ${ }^{30}$ Very surprisingly, siRNA modified with UNA only in the AS and SS overhangs, W127-W131, exhibited an even prolonged blood circulation time with siRNA species detectable $24 \mathrm{~h}$ after injection in blood and several organs such as kidney, spleen lung and hearth (Fig. 5A \& B). This was unexpected as W127-W131 is only marginally more stable than unmodified siRNA upon incubation in $80 \%$ FCS (Fig. 4B). However, W127-W131 did exhibit surprisingly high stability upon incubation in freshly prepared mouse serum (Fig. 4B). This suggests that the composition of nuclease differs between FCS and mouse serum with the latter having relatively less riboendonuclease activity. The result also underscores the necessity of testing siRNA stability in the biological fluids relevant to the particular siRNA application. Next we tested the potential of UNA modifications to enhance siRNA function in vivo. Notably, we observed a potent eGFP KD in a mice model bearing human eGFP-expressing pancreas tumor xenografts upon subcutaneous injection of the naked UNAmodified siRNA, W127-W131, whereas no effect was observed using non-modified siRNA. This demonstrates that the UNA-modified siRNA duplex was indeed biological functional and superior to unmodified siRNA (Fig. 5C) and hereby establishes UNA as an important type of chemical modification with great potential in siRNA therapeutics.

\section{Experimental}

\section{Preparation of siRNA duplexes}

The phosphoramidites were incorporated into the siRNA sequence through solid-phase DNA/RNA synthesis on an automated DNA/RNA synthesizer using O2'-TBDMS protected RNA phosphoramidites and common reagents as described in ref. 23 (for structures of chemically modified nucleotide monomers, see Fig. 1E). The oligoes are named according to the nomenclature of previously published work to allow easy comparison of results between individual studies. For siRNA annealing SS and AS were mixed in 1x Dharmacon annealing buffer (Dharmacom) and incubated at $95^{\circ} \mathrm{C}$ for $1 \mathrm{~min}$ and $1 \mathrm{~h}$ at $37^{\circ} \mathrm{C}$ and siRNA concentration was quantified using the Quant-iT RiboGreen RNA Assay Kit from Molecular Probes (Invitrogen) on a FLUOstar luminometer (BMG labtech).

\section{Cell culture, transfections and Dual-luciferase assay}

The human lung cancer cell line H1299 was grown in RPMI-1640 containing 10\% FBS, 1\% penicillin/streptomycin. A stable luciferase reporter H1299 cell line was created by transfection and subsequent pyromycin selection $\left(1 \mu \mathrm{g} \mathrm{ml} l^{-1}\right.$ medium) of H1299 cells with a psiCHECK2 vector (Promega) 
A
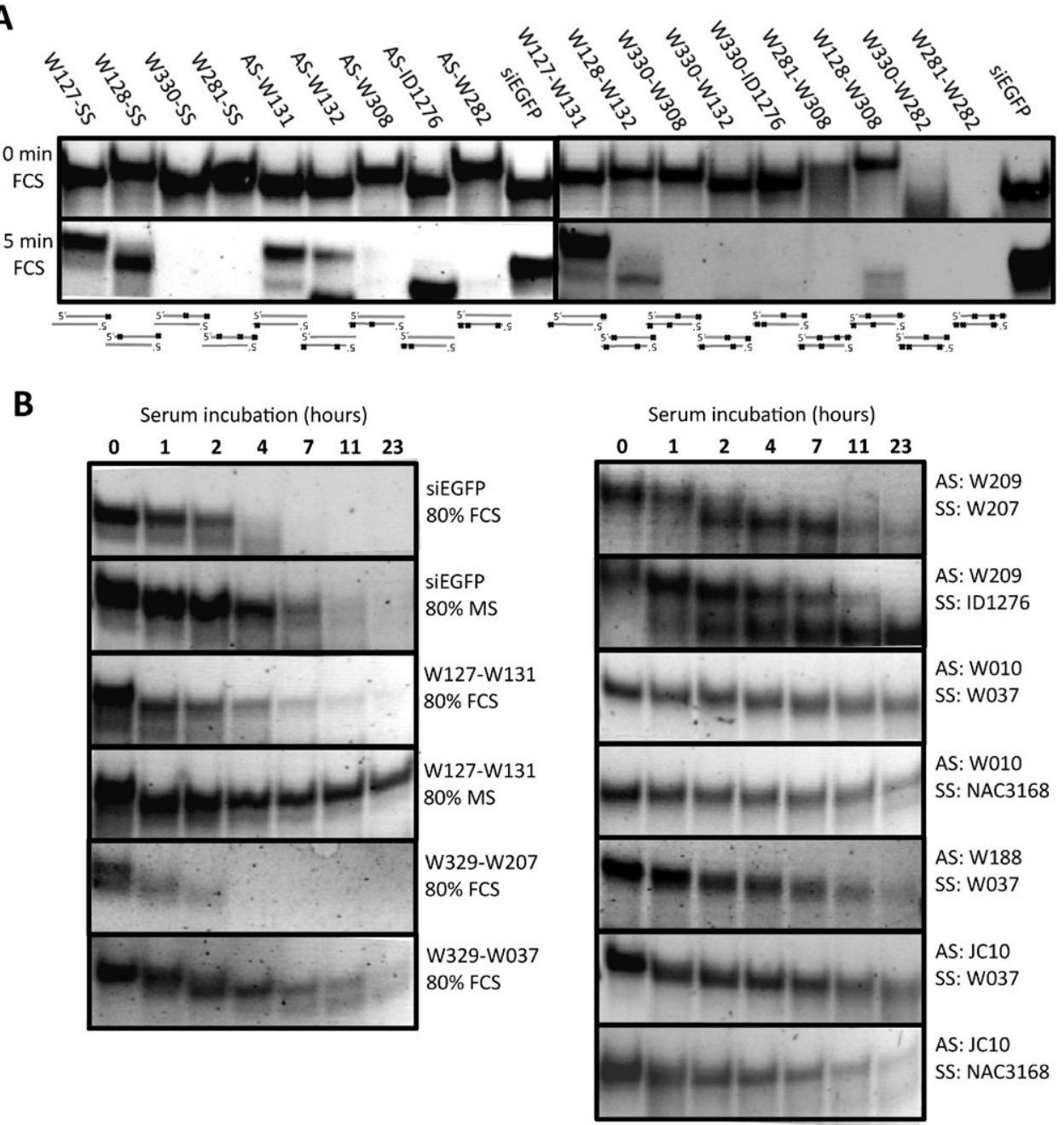

Fig. 4 Stability of UNA modified siRNAs in serum. A. Native PAGE analysis of siRNA integrity of UNA-modified AS and SS upon siRNA annealing (upper panel, no serum incubation) or after 5 min of incubation in $80 \%$ Foetal calf serum (FCS) (lower panel). The position of the UNA modifications or LNA modifications are shown schematically as black crosses (UNA) or grey lines (LNA), respectively. B. Native PAGE analysis of siRNA integrity of UNA/LNA modified siRNAs upon incubation in $80 \%$ FCS or $80 \%$ freshly prepared mouse serum (MS) for the indicated time-period.

modified to contain a perfect target site for eGFP in the multiple cloning site (MCS) and a pyromycin selection cassette. siRNA transfections were performed in triplicates using the Lipofectamine 2000 reagent (Invitrogen) in a 96 well format using 15.000 cells in $100 \mu 1$ serum-free RPMI. Growth medium was added $4 \mathrm{~h}$ after transfection and cell lysates were harvested after $48 \mathrm{~h}$ and dual-luciferase assays were performed using the 'Dual-luciferase reporter assay system' (Promega) according to the manufacturer's protocol on a FLUOstar luminometer (BMG labtech); renilla luciferase signals (sample) were normalized to the firefly luciferase signals (transfection control). Transient transfection experiments using the reporter constructs pISO-AS-target and pISO-SStarget to evaluate AS and SS efficiency (Fig. 2C) is described previously. ${ }^{5}$

\section{Evaluation of siRNA serum stability}

Annealed UNA/LNA-modified siRNAs were incubated at $37{ }^{\circ} \mathrm{C}$ in $80 \%$ fetal calf serum (FCS) or $80 \%$ freshly prepared mouse serum. Aliquots of $5 \mu \mathrm{l}$ (each containing $20 \mathrm{pmol}$ of siRNA) were diluted in $25 \mu \mathrm{l} 1.2 \times \mathrm{TBE}$ loading buffer $(1.2 \times \mathrm{TBE}, 10 \%$ glycerol, bromphenol blue) and snap-frozen on dry ice immediately upon sample taking. Samples were run on a $15 \%$ native polyacrylamide gel and stained using SYBR Gold $^{\circledR}$ (Invitrogen).

\section{Biostability of siRNAs in mice}

For investigation of siRNA biostability and biodistribution in mice $10 \mu \mathrm{g}$ unmodified, LNA- or UNA- modified siRNA duplex was administered i.v. in a total volume of $200 \mu$ l. Total RNA was purified by Trizol reagent (Invitrogen, Carlsbad, CA) from frozen blood samples and weighed tissue in RNAlater (Ambion). RNA from blood $(2 \mu \mathrm{g})$ and from tissues $(4 \mu \mathrm{g})$ was run on $15 \%$ denaturing polyacrylamide gels and transferred onto Hybond-N + membrane (Amersham Biosciences, Uppsala, Sweden). After UV crosslinking, the membranes were analyzed on a phophorimager to visualize the ${ }^{32} \mathrm{P}$ labelled AS of the siRNA. To perform northern blotting, the same 

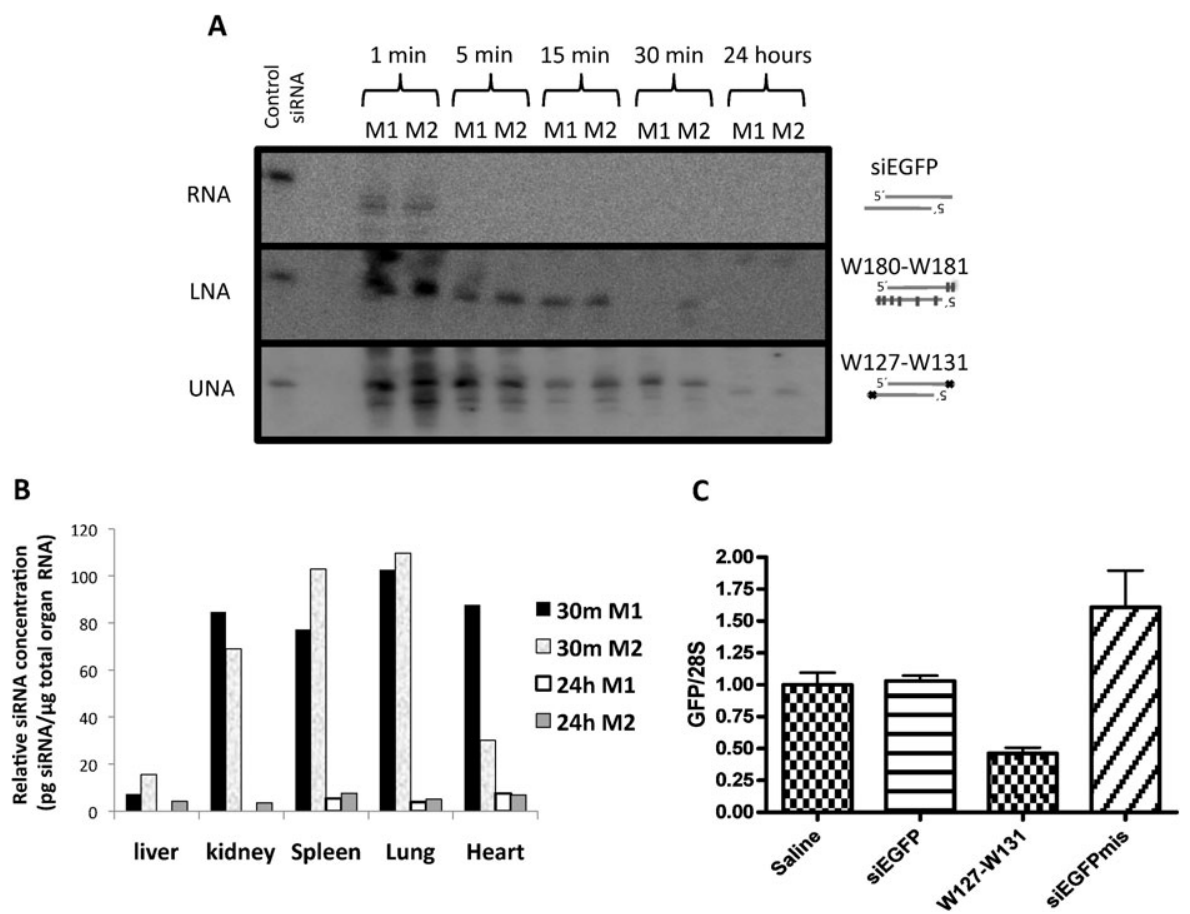

Fig. 5 Stability, distribution and activity of UNA-modified siRNAs in vivo. A. Stability of unmodified, UNA- or LNA-modified siRNA in blood upon intravenous injection in mice as evaluated by Northern blotting. Experiments were performed in duplicates and M1 and M2 denote the two mice used for the experiment. The position of the UNA modifications or LNA modifications are shown schematically as black crosses (UNA) or grey lines (LNA), respectively. B. Biodistribution of UNA-modified duplexes from various organs $30 \mathrm{~min}$ and $24 \mathrm{~h}$ after intravenous injection. Analysis of siRNA integrity was performed by Northern blotting (similar to Fig. 5A) and the siRNA levels were quantified and normalized to total RNA harvested from the specific organ. Experiments were performed in duplicates and M1 and M2 denoted the two mice used for the experiment. C. Efficiency in eGFP KD by UNA-modified siRNAs in a human pancreas cancer xenograft model in mice. siEGFP is an unmodified siRNA duplex containing the AS W053 and SS W207. siEGFPmis is a mismatch control.

filters were stored for four months (eight half-lives of 32P) and probed with [gamma-32P] adenosine triphosphate-labelled sense-strand LNA-modified siRNA according to standard procedures.

\section{In vivo efficacy of siRNAs in human xenograft liver cancer model in mice}

siRNA was tested for its ability to repress EGFP expression in vivo. Subcutaneous tumours were induced in 8- to 10-weekold NMRI nu/nu mice (Charles River, Maastricht, the Netherlands) by injection of $10^{6}$ MiaPaca II tumour cells in Matrigel (BD Biosciences). One week after tumour cell injection, when tumour take was positive, administration of the siRNA started. Administration was via osmotic minipumps (model 1007D; Alzet Corp., Palo Alto,CA) placed subcutaneously. Total RNA was isolated from the tumours and livers and EGFP mRNA levels were determined in these samples by Northern blotting.

\section{Conclusion}

Our current and previous work has identified UNA as an important modification in siRNA design. We have previously reported that UNA-modification of the SS $3^{\prime}$ overhang can enhance siRNA potency and that UNA modification of ASs could enhance target cell viability. ${ }^{5}$ Recently we have also found that UNA-modification of the AS seed region can reduce off-target effects (Bramsen et al., in prep.). Here we have extended our analysis of UNA-modification in siRNA design and describe novel strategies to improve siRNA performance. We find that single UNA modifications are well tolerated at most tested positions in the AS and SS; However, additional UNA-modifications, especially in the AS, lead to reduced silencing efficiency (Fig. 1A-C) likely by destabilizing the siRNA duplex or interactions with the target mRNA. In accordance, UNA-modification of the siRNA stem often lead to reduction in serum stability and siRNA duplexes with several UNA-modifications are not efficiently formed under standard siRNA annealing conditions (Fig. 4A). Yet, we find that even few UNA-modifications can significantly improve siRNA performance if placed strategically within the siRNA duplex; introduction of UNA in the $3^{\prime}$ end of the SS can enhance siRNA efficiency of thermodynamically asymmetric siRNA by favoring AS incorporation into RISC (Fig. 2). Furthermore, UNA modification seems well suited in siRNA designs in combination with stabilizing modifications such as e.g. LNA to enhance siRNA potency: Incorporation of UNA into an extensively LNA-modified SS enhanced the potency of ASs containing several types of chemical modifications (Fig. 3). Similarly, inserting UNA into both unmodified and extensively LNA-modified ASs enhanced siRNA potency in combination with several extensively modified SS (Fig. 3). Notably, introduction of UNA into the stem of extensively modified siRNA did not dramatically reduce serum stability in 
contrast to siRNAs modified exclusively with UNA (Fig. 4B). Thus, UNA can be used to improve the KD efficiency of heavily modified siRNA without necessarily hampering the stability of the duplex. Interestingly, we find a prolonged survival of siRNA modified by UNA in their $3^{\prime}$ overhangs upon intravenous injection in mice even when compared to extensively LNA-stabilized siRNAs (Fig. 5). Moreover, these UNA-modified siRNAs are biologically active leading to $>50 \% \mathrm{KD}$ of eGFP expression in a pancreas tumor xenograft model in mice in contrast to unmodified siRNAs. Collectively, this makes the UNA modification a potent chemical modification for increasing efficiency, stability and specificity of siRNAs with great potential in therapeutics.

\section{Acknowledgements}

This work was supported by the EU-FP6 RIGHT project (no. LSHB-CT-2004-005276) and the Danish National Research Foundation. We thank Claus Bus and Rita Rosendahl Hansen for excellent technical assistance.

\section{Contributions}

M.M.P, M.B.L, N.L, S.L.W, O.R.M and J.B.B performed research; J.B.B., J.K., J.W. designed research and J.B.B. wrote the article. Access publication charges for this article were provided by the EU-FP6 RIGHT project (no. LSHB-CT2004-005276).

\section{References}

1 J. Soutschek, A. Akinc, B. Bramlage, K. Charisse, R. Constien, M. Donoghue, S. Elbashir, A. Geick, P. Hadwiger and J. Harborth, et al., Therapeutic silencing of an endogenous gene by systemic administration of modified siRNAs, Nature, 2004, 432, 173-178.

2 D. V. Morrissey, K. Blanchard, L. Shaw, K. Jensen, J. A. Lockridge, B. Dickinson, J. A. McSwiggen, C. Vargeese, K. Bowman and C. S. Shaffer, et al., Activity of stabilized short interfering RNA in a mouse model of hepatitis B virus replication, Hepatology, 2005, 41, 1349-1356.

3 D. V. Morrissey, J. A. Lockridge, L. Shaw, K. Blanchard, K. Jensen, W. Breen, K. Hartsough, L. Machemer, S. Radka and V. Jadhav, et al., Potent and persistent in vivo anti-HBV activity of chemically modified siRNAs, Nat. Biotechnol., 2005, 23, 1002-1007.

4 S. D. Rose, D. H. Kim, M. Amarzguioui, J. D. Heidel, M. A. Collingwood, M. E. Davis, J. J. Rossi and M. A. Behlke, Functional polarity is introduced by Dicer processing of short substrate RNAs, Nucleic Acids Res., 2005, 33, 4140-4156.

5 J. B. Bramsen, M. B. Laursen, C. K. Damgaard, S. W. Lena, B. R. Babu, J. Wengel and J. Kjems, Improved silencing properties using small internally segmented interfering RNAs, Nucleic Acids Res., 2007, 35, 5886-5897.

6 S. M. Elbashir, J. Harborth, W. Lendeckel, A. Yalcin, K. Weber and T. Tuschl, Duplexes of 21-nucleotide RNAs mediate RNA interference in cultured mammalian cells, Nature, 2001, 411, 494-498.

7 E. Maniataki and Z. Mourelatos, A human, ATP-independent, RISC assembly machine fueled by pre-miRNA, Genes Dev., 2005, 19, 2979-2990.

8 J. Martinez, A. Patkaniowska, H. Urlaub, R. Luhrmann and T. Tuschl, Single-stranded antisense siRNAs guide target RNA cleavage in RNAi, Cell, 2002, 110, 563-574.

9 S. M. Hammond, S. Boettcher, A. A. Caudy, R. Kobayashi and G. J. Hannon, Argonaute2, a link between genetic and biochemical analyses of RNAi, Science, 2001, 293, 1146-1150.

10 J. Liu, M. A. Carmell, F. V. Rivas, C. G. Marsden, J. M. Thomson, J. J. Song, S. M. Hammond, L. Joshua-Tor and G. J. Hannon, Argonaute2 is the catalytic engine of mammalian RNAi, Science, 2004, 305, 1437-1441.
11 C. Matranga, Y. Tomari, C. Shin, D. P. Bartel and P. D. Zamore, Passenger-strand cleavage facilitates assembly of siRNA into Ago2-containing RNAi enzyme complexes, Cell, 2005, 123, 607-620.

12 T. A. Rand, S. Petersen, F. Du and X. Wang, Argonaute2 cleaves the anti-guide strand of siRNA during RISC activation, Cell, 2005, 123, 621-629.

13 P. J. Leuschner, S. L. Ameres, S. Kueng and J. Martinez, Cleavage of the siRNA passenger strand during RISC assembly in human cells, EMBO Rep., 2006, 7, 314-320.

14 O. R. Mook, F. Baas, M. B. de Wissel and K. Fluiter, Evaluation of locked nucleic acid-modified small interfering RNA in vitro and in vivo, Mol. Cancer Ther., 2007, 6, 833-843.

15 J. M. Layzer, A. P. McCaffrey, A. K. Tanner, Z. Huang, M. A. Kay and B. A. Sullenger, In vivo activity of nucleaseresistant siRNAs, $R N A$, 2004, 10, 766-771.

16 V. Hornung, M. Guenthner-Biller, C. Bourquin, A. Ablasser, M. Schlee, S. Uematsu, A. Noronha, M. Manoharan, S. Akira and A. de Fougerolles, et al., Sequence-specific potent induction of IFN-alpha by short interfering RNA in plasmacytoid dendritic cells through TLR7, Nat. Med., 2005, 11, 263-270.

17 A. L. Jackson, S. R. Bartz, J. Schelter, S. V. Kobayashi, J. Burchard, M. Mao, B. Li, G. Cavet and P. S. Linsley, Expression profiling reveals off-target gene regulation by RNAi, Nat. Biotechnol., 2003, 21, 635-637.

18 A. L. Jackson, J. Burchard, J. Schelter, B. N. Chau, M. Cleary, L. Lim and P. S. Linsley, Widespread siRNA "off-target" transcript silencing mediated by seed region sequence complementarity, $R N A, 2006,12,1179-1187$.

19 M. Amarzguioui, T. Holen, E. Babaie and H. Prydz, Tolerance for mutations and chemical modifications in a siRNA, Nucleic Acids Res., 2003, 31, 589-595.

20 K. Ui-Tei, Y. Naito, S. Zenno, K. Nishi, K. Yamato, F. Takahashi, A. Juni and K. Saigo, Functional dissection of siRNA sequence by systematic DNA substitution: modified siRNA with a DNA seed arm is a powerful tool for mammalian gene silencing with significantly reduced off-target effect, Nucleic Acids Res., 2008, 36, 2136-2151.

21 A. L. Jackson, J. Burchard, D. Leake, A. Reynolds, J. Schelter, J. Guo, J. M. Johnson, L. Lim, J. Karpilow and K. Nichols, et al., Position-specific chemical modification of siRNAs reduces "off-target" transcript silencing, $R N A, 2006,12,1197-1205$.

22 D. Odadzic, J. B. Bramsen, R. Smicius, C. Bus, J. Kjems and J. W. Engels, Synthesis of 2'-O-modified adenosine building blocks and application for RNA interference, Bioorg. Med. Chem., 2008, 16, 518-529.

23 J. B. Bramsen, M. B. Laursen, A. F. Nielsen, T. B. Hansen, C. Bus, N. Langkjaer, B. R. Babu, T. Hojland, M. Abramov and A. Van Aerschot, et al., A large-scale chemical modification screen identifies design rules to generate siRNAs with high activity, high stability and low toxicity, Nucleic Acids Res., 2009, 37, 2867-2881.

24 J. Vollmer, J. S. Jepsen, E. Uhlmann, C. Schetter, M. Jurk, T. Wader, M. Wullner and A. M. Krieg, Modulation of $\mathrm{CpG}$ oligodeoxynucleotide-mediated immune stimulation by locked nucleic acid (LNA), Oligonucleotides, 2004, 14, 23-31.

25 N. Langkjaer, A. Pasternak and J. Wengel, UNA (unlocked nucleic acid): a flexible RNA mimic that allows engineering of nucleic acid duplex stability, Bioorg. Med. Chem., 2009, 17, $5420-5425$.

26 M. Petersen and J. Wengel, LNA: a versatile tool for therapeutics and genomics, Trends Biotechnol., 2003, 21, 74-81.

27 D. S. Schwarz, G. Hutvagner, T. Du, Z. Xu, N. Aronin and P. D. Zamore, Asymmetry in the assembly of the RNAi enzyme complex, Cell, 2003, 115, 199-208.

28 A. Khvorova, A. Reynolds and S. D. Jayasena, Functional siRNAs and miRNAs exhibit strand bias, Cell, 2003, 115, 209-216.

29 S. Gao, F. Dagnaes-Hansen, E. J. Nielsen, J. Wengel, F. Besenbacher, K. A. Howard and J. Kjems, The effect of chemical modification and nanoparticle formulation on stability and biodistribution of siRNA in mice, Mol. Ther., 2009, 17, 1225-1233.

30 S. Z. Glud, J. B. Bramsen, F. Dagnaes-Hansen, J. Wengel, K. A. Howard, J. R. Nyengaard and J. Kjems, Naked siLNAmediated gene silencing of lung bronchoepithelium EGFP expression after intravenous administration, Oligonucleotides, 2009, 19, $163-168$. 